\title{
Biomarkers That Differentiate Benign Prostatic Hyperplasia from Prostate Cancer: A Literature Review
}

This article was published in the following Dove Press journal: Cancer Management and Research

\author{
Christopher J McNally ${ }^{1,2}$ \\ Mark W Ruddock (D) \\ Tara Moore $\mathbb{D}^{2}$ \\ Declan J McKenna $\mathbb{D}^{2}$
}

'Randox Laboratories Ltd, Crumlin, Co. Antrim BT29 4QY, Northern Ireland; ${ }^{2}$ Biomedical Sciences Research Institute, Ulster University, Coleraine BT52 ISA, Northern Ireland
Correspondence: Declan J McKenna Tel +44 2870I24356

Email dj.mckenna@ulster.ac.uk

\begin{abstract}
Prediction of prostate cancer in primary care is typically based upon serum total prostate-specific antigen (tPSA) and digital rectal examination results. However, these tests lack sensitivity and specificity, leading to over-diagnosis of disease and unnecessary, invasive biopsies. Therefore, there is a clinical need for diagnostic tests that can differentiate between benign conditions and early-stage malignant disease in the prostate. In this review, we evaluate research papers published from 2009 to 2019 reporting biomarkers that identified or differentiated benign prostatic hyperplasia $(\mathrm{BPH})$ from prostate cancer. Our review identifies hundreds of potential biomarkers in urine, serum, tissue, and semen proposed as useful targets for differentiating between prostate cancer and BPH patients. However, it is still not apparent which of these candidate biomarkers are most useful, and many will not progress beyond the discovery stage unless they are properly validated for clinical practice. We conclude that this validation will come through the use of multivariate panels which can assess the value of biomarker candidates in combination with clinical parameters as part of a risk prediction calculator. Implementation of such a model will help clinicians stratify patients with prostate cancer symptoms in primary care, with tangible benefits for both the patient and the health service.
\end{abstract}

Keywords: prostate cancer, benign prostatic hyperplasia, biomarkers, differentiation, transrectal ultrasound-guided biopsy

\section{Introduction}

Serum prostate-specific antigen (PSA) was deemed a viable tumor marker for the detection of prostate cancer (PCa) allowing clinicians to track patient response to cancer treatment. ${ }^{1}$ However, initial investigations into PCa usually involve a combination of digital rectal examination (DRE) and measurement of PSA levels. Based on these measurements a referral for Transrectal Ultrasound (TRUS)-guided biopsy may be made. Whilst PSA has helped identify many more patients with $\mathrm{PCa}$, one of the main obstacles for clinicians is to differentiate $\mathrm{PCa}$ from non-malignant conditions. One of these conditions, benign prostatic hyperplasia (BPH), can also present with raised levels of PSA. With no universally agreed way to stratify suspected cases of $\mathrm{PCa}$ to help inform diagnostic process at this point, many men undergo unnecessary biopsy or further procedures that they may not require. ${ }^{2}$

It is therefore clear that there is a need for more accurate methods to risk stratify men who present with symptoms of PCa, to prevent the over-diagnosis and unnecessary treatment of patients with benign conditions. ${ }^{3}$ Successfully implemented in 
primary care, this would greatly reduce the over-diagnosis rates of patients, as well as relieving financial and management pressures on healthcare providers. Recent research efforts suggest that this stratification is likely to be based on the identification of reliable biomarkers which can improve on the current use of PSA measurement to diagnose PCa. This review aims to present and evaluate data from studies published over the past decade that have proposed biomarkers which may be useful for differentiating between BPH and $\mathrm{PCa}$.

$\mathrm{BPH}$ is a non-malignant enlargement of the prostate caused by cellular hyperplasia that occurs within the transitional zone. ${ }^{3} \mathrm{BPH}$ is associated with age, with around $50 \%$ of men aged $50,70 \%$ of men aged 70 , and $90 \%$ of men aged 80 being affected. ${ }^{4,5}$ The proliferation of prostatic cells leads to an increase in the size of the prostate as well as urethral obstruction and lower urinary tract infections (LUTS). Risk factors for BPH include age, decreased testicular function, metabolic syndrome, family history of BPH and obesity. ${ }^{4}$ Several studies have investigated an association between $\mathrm{BPH}$ and $\mathrm{PCa}$, although the underlying pathophysiology between the two conditions remains unclear. ${ }^{6-8}$ A meta-analysis of 19 studies involving 15,899 patients determined that $\mathrm{BPH}$ was associated with an increased risk of PCa, risk ratio (RR) 2.93 , (95\% $\mathrm{CI}=1.88-4.56), P<0.0000 .{ }^{9}$ The authors demonstrated that the association between $\mathrm{BPH}$ and $\mathrm{PCa}$ was stronger within Asian populations when compared to Caucasians; RR 6.09 and 1.54, respectively. The authors also suggested that hormones, inflammation, and metabolic syndrome likely play a role in the pathophysiology of $\mathrm{BPH} .{ }^{9}$ There is also evidence that the homeostasis between prostate cell proliferation and cell death supported by dihydrotestosterone (DHT) and estrogen is often disrupted in BPH patients. ${ }^{10}$ Additionally, aggressive BPH is associated with an elevated risk of developing $\mathrm{PCa}$ and the subsequent cancer can be high grade compared to individuals without fast-growing BPH. ${ }^{11}$ Although the majority of findings are hypothesis-generating rather than hypothesis-confirming, the evidence does support the theory that $\mathrm{BPH}$ is a risk factor involved in the pathogenesis of PCa. ${ }^{12-14}$

However, despite this evidence, it is not inevitable that $\mathrm{BPH}$ will progress to $\mathrm{PCa}$ in any given individual. Therefore, it is important to be able to distinguish BPH patients at an early stage to prevent further invasive and unnecessary tests in these individuals. PSA testing alone is not able to make this differentiation, so new biomarkers are required to improve the risk-stratification of patients at this stage.

\section{Prostate Specific Antigen}

Currently, PSA is one of the most widely used biomarkers for the detection and management of PCa. Prior to PSA measurement, PCa was mainly predicted by DRE. However, DRE as a diagnostic tool has low sensitivity and specificity, as well as user subjectivity between clinicians performing the examination. ${ }^{15}$ PSA testing was a clear diagnostic improvement and was introduced in the United States in 1987 to determine if patients were responding to curative therapy. Soon after, PSA was used for screening patients at risk of $\mathrm{PCa}$, leading to an increase in disease detection and a decline in mortality. ${ }^{16}$ PSA is a kallikrein-like serine protease produced by the epithelial cells of the prostate to help liquefy ejaculate and aid sperm motility. Extraprostatic production of PSA is provided mainly by the periurethral glands, which in turn leads to measurable levels of PSA in the serum ${ }^{17}$ The San Diegobased company Hybritech Inc. was the first company to propose a serum PSA threshold of $4.0 \mathrm{ng} / \mathrm{mL}$ after a study on healthy men. ${ }^{17}$ Subsequently, a PSA value of $>4.0 \mathrm{ng} /$ $\mathrm{mL}$ became the industry standard for recommending a prostate biopsy. Since the introduction of this threshold, studies have shown that PSA testing has a sensitivity of $67-80 \%$ and has helped diagnose a large number of patients with PCa since it was first introduced. ${ }^{17}$

However, although PSA is organ-specific, it is not a cancer-specific biomarker. BPH and other conditions, for example, prostatitis, inflammation of the prostate, can raise serum PSA levels. Conversely, PCa has been shown in males who present with normal PSA levels. ${ }^{16}$ Hence, there is a lack of specificity with PSA which can lead to over-diagnosis of $\mathrm{PCa}$ and unnecessary treatment. Data gathered from the Surveillance, Epidemiological and End Results (SEER) registry estimates that screening for PCa using PSA has resulted in $28 \%$ of over-diagnosed cases in the USA. ${ }^{18}$ Likewise, the European Randomised Study of Screening for Prostate Cancer (ERSPC) trial estimated that using PSA as a screening tool for $\mathrm{PCa}$ led to $50 \%$ of patients being overdiagnosed. ${ }^{19}$ Actively diagnosing a clinically insignificant tumor can lead to unnecessary treatment, such as radical prostatectomy or radiotherapy. To avoid this, healthcare providers are utilizing the strategy of active surveillance, where regular PSA and DRE testing is used alongside biopsies over a period of time to minimize the risk of over-diagnosis. However, regular check-ups and repeated prostate biopsies 
are invasive and can be very painful for the patient. This unsurprisingly can cause high levels of anxiety and stress and may discourage the patient to seek medical attention at all. ${ }^{16}$

It is known that serum PSA levels increase with age. This is most likely due to the contribution of an enlarged prostate associated with old age as well as the decreased retention of the prostatic epithelium. Age-specific PSA has been shown to increase the detection of $\mathrm{PCa}$ in younger men (50-59 years) by $15 \%$ but also shown to increase the number of biopsies performed by $45 \%{ }^{20}$ To address these issues, various studies have investigated more nuanced measurements of PSA expression to help improve its usefulness for PCa diagnosis. Significant research has been performed on free PSA, rather than total PSA (tPSA), demonstrating the ratios of free-to-total PSA in serum may improve the diagnostic specificity by $15-20 \%{ }^{21}$ This type of test is recommended for patients who present with PSA levels within the "grey-zone" of 4.0 to $10.0 \mathrm{ng} /$ $\mathrm{mL}$. Initially, it was proposed that high levels of free PSA were associated with benign prostate tissue and a decreased probability of $\mathrm{PCa}^{21}$ However, this test was not widely implemented as a screening tool due to inconsistencies in later studies. ${ }^{17}$ The [-2] isoform of proPSA has emerged as a promising biomarker due to its ability to differentiate between $\mathrm{PCa}$ and $\mathrm{BPH}$, where levels appear raised in $\mathrm{PCa}$. One large prospective study of patients with PCa showed that the percentage of $[-2]$ proPSA improved the specificity to $44.9 \%$ in comparison to total and free PSA which was $30.8 \%$ and $34.6 \%$ respectively whilst also achieving a sensitivity of $80 \%$ for detecting PCa. ${ }^{22}$

The Prostate Health Index (PHI) can also be considered a biomarker, calculated using the following formula: $\mathrm{PHI}=$ $([-2]$ proPSA/freePSA $) \times \sqrt{ }$ PSA. In one study, both the $[-2]$ proPSA $(\mathrm{AUC}=0.76)$ and the PHI test $(\mathrm{AUC}=0.77)$ outperformed the tPSA test when used to detect PCa between the ranges of $2.5-10 \mathrm{ng} / \mathrm{mL}^{23}$ These studies have shown the predictive superiority of these two tests compared to that of just tPSA based screening alongside a significant improvement in accuracy. However, other studies do not agree with these results and indicated that when the goal is to detect at least $95 \%$ of the aggressive tumors, PHI does not seem to be much more effective than the \%free PSA and the PSA density. ${ }^{24}$

PSA density is calculated as the tPSA in $\mathrm{ng} / \mathrm{mL}$ divided by prostate volume $(\mathrm{mL})$. Nordström et al in 2018 suggested that PSA density might inform clinicians more on biopsy decisions after determining that a cut-off of $0.10 \mathrm{ng} / \mathrm{mL}^{2}$ resulted in a detection rate of $77 \%$ of
Gleason score $\geq 7$ tumors compared to tPSA alone, 64\% $(\mathrm{n}=947){ }^{25}$ Additionally, both Verma et al in 2014 and Sebastianelli et al in 2019 both suggested that PSA density could be used to reduce unnecessary biopsies after determining the marker was significant for the detection of aggressive $\mathrm{PCa}{ }^{26,27}$ However, Liu et al in 2015 concluded in their paper that if a patient has PSA levels of $<2.0 \mathrm{ng} /$ $\mathrm{mL}$, PSA density does not differentiate the PCa effectively enough $(\mathrm{n}=343)$. $^{28}$

PSA velocity is the measure of the rate of PSA increase over time. Serum PSA velocity increases significantly in the presence of a prostate tumor compared to that of a benign disease alone. However, no evidence was found to support the recommendation that men with high PSA velocity should be biopsied in the absence of other indications. $^{29}$

Despite these varied approaches to PSA measurement, there are still many limitations to current PSA testing for PCa. The PSA test possesses a negative benefit-to-harm ratio based on population-based estimates. ${ }^{16}$ The future of individualized PSA-based screening seems to lie as a component of multivariate risk stratification, carried out by using various nomograms and prediction risk tools. ${ }^{16}$ Therefore, the current challenge is to identify other biomarkers that can be used in combination with PSA in primary care to differentiate $\mathrm{BPH}$ from $\mathrm{PCa}$.

\section{Transrectal Ultrasound-Guided Biopsy}

Grey-scale TRUS-guided biopsy is the gold standard for prostate imaging and is essential for achieving a histological diagnosis of a prostatic carcinoma through a guided biopsy. ${ }^{30}$ The TRUS-guided biopsy is also the main procedure recommended by the National Institute for Health and Care Excellence (NICE) guidelines to diagnose $\mathrm{PCa}^{31}$ Although the procedure is considered safe, the number of post-biopsy complications are on the rise, and often reported in up to $50 \%$ of cases. Complications include pain, haematuria, haematospermia, urinary retention as well as infection. ${ }^{32}$ The pain reported from a TRUS-guided biopsy is relative to the number of cores removed. ${ }^{33}$ In a study performed on patients after a sextant biopsy (six-core removal), $94 \%$ of patients found the procedure painful and $24 \%$ of patients reported the pain as moderate to severe. The extended 12-core biopsy can result in higher levels of pain, subsequent inadequate sampling and eventual abandonment of the procedure. ${ }^{33}$ Many patients have refused when asked if they would undergo a repeat prostate biopsy. Pain is subjective, it is difficult to quantify. However, it is clear that both psychosocial factors and physical attributes 
play an important role when patients require a prostate biopsy. ${ }^{34}$ Aside from immediate surgical pain, complications and infection can occur. UTIs were reported at an occurrence rate of $6 \%$ post-TRUS biopsy, with $30-50 \%$ of patients within that $6 \%$ going on to develop bacteremia. Approximately $0.1 \%$ $-2.2 \%$ of TRUS biopsy patients will develop severe sepsis. ${ }^{35}$ One study reported that 1 in 4 post-TRUS biopsy patients hospitalized due to $E$. coli bacteremia had severe sepsis that required them to be admitted to the intensive care unit (ICU). ${ }^{32}$ Interestingly, there are reports to suggest that the rate of infectious complications after TRUS biopsy is on the rise. A study based in Ontario, Canada reported an increase in the rate of hospitalization within 30 days following a TRUS biopsy, from $1.0 \%$ of patients in 1996 to $4.1 \%$ in $2005(\mathrm{P}<$ $0.0001) .^{36}$

Although the frequency of complications is relatively low, the problem is still a substantial one, given the number of biopsies performed and the associated economic burden from treating biopsy-related complications. ${ }^{32}$ A new test that has high specificity and could confidently stratify patients into groups that require a biopsy and those who could be stratified to watch and wait, would significantly reduce the number of post-TRUS biopsy complications.

The rapidly growing use of multiparametric magnetic resonance imaging (mp-MRI) offers a much safer alternative to the TRUS-guided biopsy. The use of MRI continues to show increased accuracy for the detection, localization, risk stratification and staging of PCa for patients. ${ }^{37-40}$ The largest benefit will come from the reduced number of unnecessary biopsies being performed, which in turn will significantly reduce levels of overdiagnosis within patients displaying signs of $\mathrm{PCa}{ }^{41}$ However, although mp-MRI has shown promising results, it is still not perfect. There is a risk that 5-20\% of index lesions are missed. However, in combination with standard TRUS-guided biopsy, this can be improved. ${ }^{42-44}$ Additionally, current MRI technology also lacks the resolution to detect tumors with a smaller volume and a lower Gleason score, making it less reliable at detection early-stage $\mathrm{PCa} .{ }^{43,44}$ Nevertheless, the benefits of mp-MRI are clear and offer an attractive alternative to TRUS-guided biopsies, although access to the required instrumentation and resources may still be a clinical barrier to widespread implementation.

\section{The Search for New Biomarkers}

The most ideal biomarkers are ones that can be measured accurately and reproducibly in a minimally invasive manner. ${ }^{45}$ Biomarkers can be measured in tissue, blood, urine and/or semen, but standardized collection and analysis of these samples can present some unique challenges. As a result, there is no consensus on the best samples to use, or the optimal biomarkers to measure once the sample has been collected. This review aims to provide a comprehensive overview of research being performed that purport to identify genomic and/or proteomic biomarkers that can differentiate between $\mathrm{BPH}$ and PCa. The following search criteria were employed:

- Article obtained using either PubMed or Google Scholar

- Manuscripts published within the last 10 years

- Articles are in English

- Biomarkers must be analyzed from either blood, urine, tissue or seminal fluid

- Analyzed specifically and separately both PCa and BPH specimens in the study

- Attempted to differentiate between PCa and BPH

Using this filtered approach, we retrieved 104 published papers for blood and urine-based biomarkers. Their findings are reported in Tables 1-4, with similar data for tissue and seminal fluid biomarkers gathered from 49 published papers presented in Supplementary Tables 1-3.

\section{Blood-Based Biomarkers Genomic Blood Biomarkers}

Table 1 provides a list of blood-based biomarkers from 19 papers that have been investigated by researchers using genomic techniques to identify patterns that could potentially differentiate $\mathrm{BPH}$ and $\mathrm{PCa}$.

\section{Proteomic Blood Biomarkers}

Table 2 provides a list of blood-based biomarkers from 44 papers that have been investigated by researchers using proteomic techniques to identify patterns that could potentially differentiate $\mathrm{BPH}$ and $\mathrm{PCa}$.

\section{Urine-Based Biomarkers Genomic Urine Biomarkers}

Table 3 provides a list of urine-based biomarkers from 21 papers that have been investigated by researchers using genomic techniques to identify patterns that could potentially differentiate $\mathrm{BPH}$ and $\mathrm{PCa}$.

\section{Proteomic Urine Biomarkers}

Table 4 provides a list of urine-based biomarkers from 23 papers that have been investigated by researchers using 
Table I Blood-Based Biomarkers Derived from Genomic Techniques

\begin{tabular}{|c|c|c|c|c|c|}
\hline Biomarkers & $\begin{array}{l}\text { BPH } \\
\text { (n) }\end{array}$ & $\begin{array}{l}\mathrm{PCa} \\
(\mathbf{n})\end{array}$ & Method & $\begin{array}{l}\text { All Significant } \\
(p<0.05)\end{array}$ & Reference \\
\hline miR-I5a $\downarrow$ & \multirow[t]{3}{*}{35} & \multirow[t]{3}{*}{35} & \multirow[t]{3}{*}{ RT-PCR } & \multirow[t]{3}{*}{ Yes } & \multirow[t]{3}{*}{46} \\
\hline 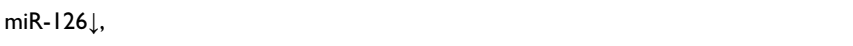 & & & & & \\
\hline 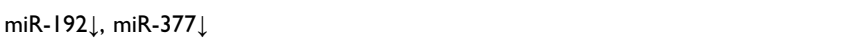 & & & & & \\
\hline $\mathrm{miR}-18 \mathrm{a} \uparrow$ & 24 & 24 & RT-PCR & Yes & 47 \\
\hline let-7c $\downarrow$, let-7e $\downarrow$, & \multirow[t]{6}{*}{60} & \multirow[t]{6}{*}{64} & \multirow[t]{6}{*}{$\mathrm{qPCR}$} & \multirow[t]{6}{*}{ Yes } & \multirow[t]{6}{*}{48} \\
\hline let-7i $\downarrow$, & & & & & \\
\hline miR-26a-5p $\downarrow$ & & & & & \\
\hline miR-26b-5p $\downarrow$ & & & & & \\
\hline miR-18b-5p $\downarrow$ & & & & & \\
\hline miR-25-3p $\downarrow$ & & & & & \\
\hline Retinoic Acid Receptor $\beta 2$ (RAR $\beta 2) \uparrow$ & 94 & 91 & qPCR & Yes & 49 \\
\hline Cell-Free DNA $\uparrow$ & 112 & 96 & qPCR & Yes & 50 \\
\hline let-7a $\uparrow, \operatorname{miR}-210 \uparrow, \operatorname{miR}-562 \uparrow, \operatorname{miR}-616 \uparrow$ & 13 & 31 & RT-PCR & Yes & 51 \\
\hline let-7c $\downarrow$, miR-30c $\downarrow$, & \multirow[t]{3}{*}{16} & \multirow[t]{3}{*}{59} & \multirow[t]{3}{*}{ qPCR } & \multirow[t]{3}{*}{ Yes } & \multirow[t]{3}{*}{52} \\
\hline mirR-|4| & & & & & \\
\hline miR-375 $\downarrow$ & & & & & \\
\hline miR-708\, & \multirow[t]{7}{*}{39} & \multirow[t]{7}{*}{76} & \multirow[t]{7}{*}{ qPCR } & \multirow[t]{7}{*}{ Yes } & \multirow[t]{7}{*}{53} \\
\hline $\operatorname{miR}-22 I \downarrow$ & & & & & \\
\hline miR-518d-5p $\downarrow$ & & & & & \\
\hline $\operatorname{miR}-675 \uparrow$ & & & & & \\
\hline miR-III80个, & & & & & \\
\hline 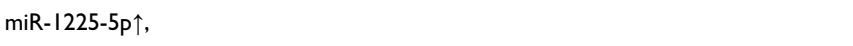 & & & & & \\
\hline $\operatorname{miR}-659 \uparrow$ & & & & & \\
\hline $\operatorname{miR}-26 \mathrm{a} \uparrow$ & \multirow[t]{2}{*}{18} & \multirow[t]{2}{*}{37} & \multirow[t]{2}{*}{ qPCR } & \multirow[t]{2}{*}{ Yes } & \multirow[t]{2}{*}{54} \\
\hline $\operatorname{miR}-195 \uparrow$, let $7 i \uparrow$ & & & & & \\
\hline Glutathione S-Transferase Pi I (GSTPI) $\uparrow$ & 34 & 31 & MS-PCR & Yes & 55 \\
\hline miR-499, & \multirow[t]{3}{*}{353} & \multirow[t]{3}{*}{355} & \multirow[t]{3}{*}{ PCR-RFLP } & \multirow[t]{3}{*}{ Yes } & 56 \\
\hline miR-196a2, & & & & & \\
\hline miR-27a variants & & & & & \\
\hline Growth Arrest and DNA Damage Inducible Alpha (GADD45a) Methylation $\uparrow$ & 48 & 34 & PyroSequencing & Yes & 57 \\
\hline 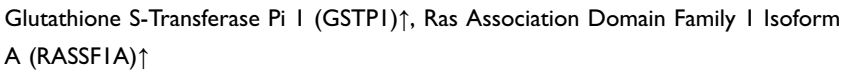 & 103 & 83 & MS-PCR & Yes & 58 \\
\hline Cell-Free DNA $\uparrow$ & 76 & 50 & Spectrophotometry & Yes & 59 \\
\hline $\operatorname{miR}-375 \uparrow$ & 35 & 146 & $\mathrm{qPCR}$ & Yes & 60 \\
\hline
\end{tabular}


Table I (Continued).

\begin{tabular}{|c|c|c|c|c|c|}
\hline Biomarkers & $\begin{array}{l}\text { BPH } \\
\text { (n) }\end{array}$ & $\begin{array}{l}\mathrm{PCa} \\
(\mathrm{n})\end{array}$ & Method & $\begin{array}{l}\text { All Significant } \\
(p<0.05)\end{array}$ & Reference \\
\hline $\operatorname{miR}-4 \mid 0-5 p \uparrow$ & 121 & 149 & qPCR & Yes & 61 \\
\hline $\begin{array}{l}\text { SAP30L Antisense RNA I (SAP30L-ASI) } \downarrow \text {, SWI/SNF Complex Antagonist Associated } \\
\text { With Prostate Cancer I (SChLAPI) } \uparrow\end{array}$ & 46 & 34 & qPCR & Yes & 62 \\
\hline MD-miniRNA $\uparrow$ & 32 & 63 & qPCR & Yes & 63 \\
\hline miR-I5a $\downarrow$, miR- $\mid 6-I \downarrow$ & 70 & 70 & qPCR & Yes & 64 \\
\hline
\end{tabular}

Note: $\downarrow / \uparrow$ : Expression levels in PCa group compared to that of BPH group.

Abbreviations: miRNA, microRNA, Let-7, lethal-7 gene family, RT-PCR, reverse transcription-polymerase chain reaction, qPCR, quantitative polymerase chain reaction, MS-PCR, methylation specific-polymerase chain reaction, PCR-RFLP, polymerase chain reaction-restriction fragment length polymorphism.

proteomic techniques to identify patterns that could potentially differentiate BPH and PCa.

\section{Tissue- and Semen-Based Biomarkers}

$\underline{\text { Supplementary Tables } 1}$ and $\underline{2}$ provide a list of tissuebased genomic and proteomic biomarkers, respectively, retrieved from 46 published papers, which are proposed as differentiating between BPH and PCa.

Supplementary Table 3 provides a list of biomarkers from seminal fluid retrieved from 3 published papers which are proposed as differentiating between $\mathrm{BPH}$ and $\mathrm{PCa}$.

\section{Discussion}

The data presented demonstrate clear global research interest in finding biomarkers that can differentiate between $\mathrm{PCa}$ and BPH. It is also notable that major advances in both genomic and proteomic technologies have helped facilitate the identification and analysis of many novel biomarkers. The challenge now is to determine which biomarkers, or combination of biomarkers, provides the most effective way to risk stratify PCa patients in primary care. This will require careful, robust analysis to ensure the most useful candidates are selected.

It is interesting to note that the majority of biomarkers listed are all different, with very little overlap between different groups investigating the same biomarkers. One set of biomarkers that did receive attention from multiple groups were PSA glycoforms, again emphasizing the importance of PSA-related measurements for an accurate diagnosis. This is an umbrella term reflecting the various glycosylation profiles that PSA can possess, yet very few of the same PSA glycoforms were investigated by separate groups. The investigators used carbohydrate-binding proteins called lectins to detect these glycolytic changes in an immunoassay format. ${ }^{152}$ All of the groups investigating various PSA glycoforms reported successful results in some form in both serum and urine. However, within other cancers, lectins typically only detected late-stage malignancies and a select few of the papers referenced here that reported noteworthy results did not supply the Gleason score. Hence, PSA-related measurements remain likely to be an important factor for PCa prediction and biopsy referral, but the information contained in the tables demonstrate that many other candidate biomarkers offer clear potential for improving $\mathrm{PCa}$ diagnosis, prognosis, and management. For example, Filamin-A and Filamin$\mathrm{B}$ are mentioned in our tables but are also well cited as significant contributors to the differentiation of $\mathrm{PCa}$ from non-cancer patients. The two proteins play a major role in cell migration, vascular development, extracellular signaling and activity of integrins. ${ }^{95,153}$ Filamin-A and androgen receptor (AR) association play a role in nerve growth factor (NGF) induced cell migration where it is known PCa is associated with the synthesis of large amounts of NGF which then stimulates tyrosine receptor kinase A (TrkA). ${ }^{154}$ This is just one example of potential successful PCa biomarkers that have the possibility of identifying cancer quicker or preventing overdiagnosis within patients.

However, lack of follow-up in terms of validation and clinical trials means many of these candidate biomarkers will not progress beyond the discovery stage. Research efforts need to be improved in terms of validating these candidate biomarkers within larger cohorts to translate the findings to clinical practice. ${ }^{155}$ Moreover, the implementation of improved risk stratification approaches for $\mathrm{PCa}$ 
Table 2 Blood-Based Biomarkers Derived from Proteomic Techniques

\begin{tabular}{|c|c|c|c|c|c|}
\hline Biomarkers & $\begin{array}{l}\text { BPH } \\
\text { (n) }\end{array}$ & $\begin{array}{l}\mathrm{PCa} \\
\text { (n) }\end{array}$ & Method & $\begin{array}{l}\text { All } \\
\text { Significant } \\
(p<0.05)\end{array}$ & Reference \\
\hline Total Cholesterol $\uparrow$, Triglycerides $\uparrow$ & 40 & 40 & $\begin{array}{l}\text { Atomic Absorption } \\
\text { Spectrophotometry }\end{array}$ & Yes & 65 \\
\hline Free to Total PSA Ratio $\downarrow$ & 283 & 49 & Immunoassay & Yes & 66 \\
\hline $\begin{array}{l}\text { Pigment Epithelium-Derived Factor (PEDF) } \downarrow \text {, Zinc- } \alpha 2 \text {-Glycoprotein } \\
\text { (ZAG) }\end{array}$ & 13 & 37 & $\begin{array}{l}\text { 2D-DIGE, MS, WB, ELISA, } \\
\text { IHC }\end{array}$ & No & 67 \\
\hline $\begin{array}{l}\text { Glutathione peroxidase } 3 \text { (GPx-3) } \downarrow \text {, Apolipoprotein A-IV (ApoA-IV) } \\
\downarrow \text {, ApoA-I } \downarrow \text {, Coagulation Factor XIII B Chain } \uparrow \text {, Antithrombin-III } \downarrow, \alpha \text { - } \\
\text { I-Antitrypsin } \downarrow, \alpha-2 \text {-Macroglobin } \downarrow \text {, Thrombin } \downarrow \text {, Kininogen-I } \uparrow\end{array}$ & 14 & 32 & $\begin{array}{l}\text { 2D-DIGE, MS, NMR, } \\
\text { Gene Ontology } \\
\text { Enrichment Analysis }\end{array}$ & Yes & 68 \\
\hline Matrix Metalloproteinase-26 (MMP26) $\uparrow$ & 40 & 80 & ELISA & Yes & 69 \\
\hline Cyclin BI $\uparrow$ & 21 & 174 & ELISA, WB & Yes & 70 \\
\hline Prostate Health Index $(\mathrm{PHI}) \uparrow, \alpha 2,3-$ Sialylated PSA $\uparrow$ & 29 & 50 & $\begin{array}{l}\text { Glycosylation } \\
\text { Immunoassay }\end{array}$ & Yes & 71 \\
\hline 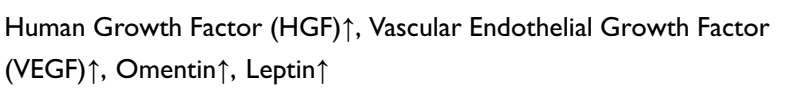 & 40 & 40 & ELISA & Yes & 72 \\
\hline Prostate Health Index $(\mathrm{PHI}) \uparrow$ & 150 & 113 & Immunoassay & Yes & 73 \\
\hline$\alpha \mathrm{l}, 2$-Fucosylated PSA $\uparrow$ and $\beta$-N-Acetylgalactosaminylated $\mathrm{PSA} \uparrow$ & 20 & 20 & $\begin{array}{l}\text { Lectin Column } \\
\text { Chromatography, ELISA }\end{array}$ & Yes & 74 \\
\hline Estradiol $\uparrow$, Insulin $\downarrow$, Insulin Growth Factor I (IGF-I) $\downarrow$ & 70 & 70 & Immunoassay & Yes & 75 \\
\hline$\beta$-N-Acetylgalactosaminylated PSA $\uparrow$ & 184 & 244 & Immunoassay & Yes & 76 \\
\hline $\begin{array}{l}\text { Apolipoprotein A2 (APOA2) } \downarrow \text {, Complement C3 Chain Fragment } \\
\text { (C3f) } \downarrow \text {, Inter-Alpha-Trypsin Inhibitor Heavy Chain }\end{array}$ & \multirow[t]{2}{*}{8} & \multirow[t]{2}{*}{8} & \multirow[t]{2}{*}{$\begin{array}{l}\text { 2DE SS, WB, LA } \\
\text { Chromatography, MS }\end{array}$} & \multirow[t]{2}{*}{ Yes } & \multirow[t]{2}{*}{77} \\
\hline 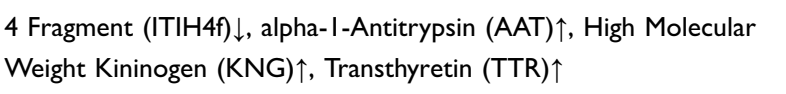 & & & & & \\
\hline tPSA $\uparrow$, Carbonic & \multirow[t]{2}{*}{120} & \multirow[t]{2}{*}{100} & \multirow[t]{2}{*}{ Piezoelectric Assay } & \multirow[t]{2}{*}{ Yes } & \multirow[t]{2}{*}{78} \\
\hline Anhydrase I (CAI) $\uparrow$, IL-6 Soluble Receptor (IL-6sR) $\downarrow$, Spondin- $2 \downarrow$ & & & & & \\
\hline Platelet to Lymphocyte Ratio $\uparrow$ & 110 & 76 & Cell Count & No & 79 \\
\hline Prolidase, Malondialdehyde, Superoxide Dismutase & 51 & 30 & Spectrophotometry & No & 80 \\
\hline 60S Ribosomal Protein L7 Clones $\uparrow$ & 70 & 49 & Protein Macroarrays & Yes & 81 \\
\hline Glypican-I $\downarrow$ & 15 & 15 & ELISA, FC, WB & Yes & 82 \\
\hline f/tPSA Ratio $\downarrow, \alpha 2,3$-Sialylated PSA $\uparrow$, Cathepsin D $\uparrow$ & 100 & 75 & ELISA & Yes & 83 \\
\hline $\begin{array}{l}\text { Secreted group IIA phospholipase A2 (sPLA2-IIA), C-reactive } \\
\text { protein (CRP) }\end{array}$ & 25 & 25 & ELISA & No & 84 \\
\hline $\begin{array}{l}\text { Serum Amyloid A (SAA), Secreted Group IIA Phospholipase A2 } \\
\text { (sPLA2-IIA), C-Reactive Protein (CRP) }\end{array}$ & 55 & 55 & Immunoassay & No & 85 \\
\hline sPSP94/tPSA Ratio $\uparrow$ & 44 & 33 & ELISA & Yes & 86 \\
\hline
\end{tabular}


Table 2 (Continued).

\begin{tabular}{|c|c|c|c|c|c|}
\hline Biomarkers & $\begin{array}{l}\text { BPH } \\
\text { (n) }\end{array}$ & $\begin{array}{l}\mathrm{PCa} \\
(\mathrm{n})\end{array}$ & Method & $\begin{array}{l}\text { All } \\
\text { Significant } \\
(p<0.05)\end{array}$ & Reference \\
\hline$\alpha 2,3$-Sialylated PSA $\uparrow$ & 35 & 35 & $\begin{array}{l}\text { Lectin Column } \\
\text { Chromatography, ELLA }\end{array}$ & Yes & 87 \\
\hline$\alpha \mathrm{I}, 2$ Fucosylated PSA $\uparrow$ & 13 & 13 & ELLA & Yes & 88 \\
\hline Dihydrotestosterone (DHT) $\downarrow$ & 97 & 60 & LC-MS/MS, IHC & Yes & 89 \\
\hline 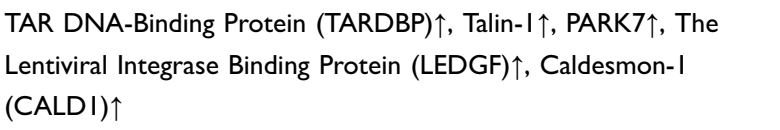 & 39 & 41 & Microarrays & Yes & 90 \\
\hline Alcohol Dehydrogenase Isoenzyme II (ADH II) & 34 & 52 & Spectrophotometry & Yes & 91 \\
\hline Cluster of differentiation 40 (CD40L) $\downarrow$ & 15 & 15 & ELISA & Yes & 92 \\
\hline Mac-2 Binding Protein (Mac-2BP) & 50 & 50 & ELISA & No & 93 \\
\hline $\begin{array}{l}\text { Serum Insulin-Like Growth Factor I, Insulin-Like Growth Factor } \\
\text { Binding Protein } 3 \text { (IGFI, IGFBP3) }\end{array}$ & 113 & 36 & $\begin{array}{l}\text { ELISA, } \\
\text { Radioimmunoassays }\end{array}$ & No & 94 \\
\hline Filamin A, Filamin B, Keratin-19 (FLNA, FLNB and KRTI9) & 122 & 311 & ELISA & Yes & 95 \\
\hline $\begin{array}{l}\text { Fibronectin I } \uparrow, \text { Afamin } \uparrow, \alpha-2 \text {-HS-Glycoprotein Chain B, } \\
\text { Ceruloplasmin } \uparrow, \beta-2 \text {-glycoprotein I } \uparrow\end{array}$ & 5 & 5 & iTRAQ, IHC, WB, MS & Yes & 96 \\
\hline PSA 2-DE Subform F3 $\downarrow$ & 20 & 20 & $\begin{array}{l}\text { ELISA, } \\
\text { Immunoadsorption, 2-DE, } \\
\text { Immunodetection }\end{array}$ & Yes & 97 \\
\hline Prostate Specific Antigen (PSA)^, Prostatic Acid Phosphatase (PAP) $\downarrow$ & 30 & 24 & ELISA, Kinetic Method & Yes & 98 \\
\hline Vascular Endothelial Growth Factor (VEGF)^ & 57 & 44 & ELISA & Yes & 99 \\
\hline tPSA $\uparrow$, Mean Platelet Volume $\downarrow$, and Platelet Distribution Width $\uparrow$ & 108 & 100 & $\begin{array}{l}\text { Immunoassay, Flux } \\
\text { Cytometry }\end{array}$ & Yes & 100 \\
\hline Fetuin $\uparrow$ & - & - & $\begin{array}{l}\text { Nanoelectrode Label Free } \\
\text { Detection }\end{array}$ & Yes & 101 \\
\hline 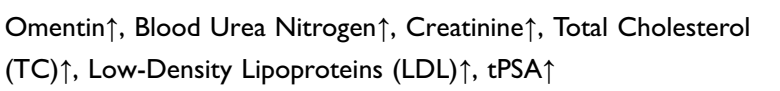 & 30 & 50 & $\begin{array}{l}\text { ELISA, } \\
\text { Spectrophotometry }\end{array}$ & Yes & 102 \\
\hline 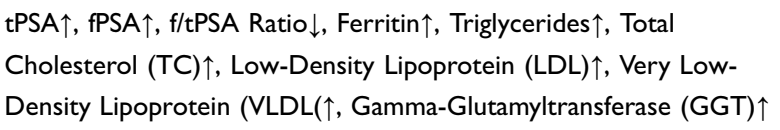 & 951 & 2002 & $\begin{array}{l}\text { Immunoassay, } \\
\text { Spectrophotometry }\end{array}$ & Yes & 104 \\
\hline Claudin $3 \uparrow$ & 69 & 15 & MS & Yes & 105 \\
\hline Testosterone/Prostate-Specific Antigen ratio (T/PSA) $\downarrow$ & 92 & 164 & Immunoassay & Yes & 106 \\
\hline Serum PF4VI $\downarrow$, tPSA $\uparrow$ & 38 & 66 & iTRAQ, IHC, WB, ELISA & Yes & 107 \\
\hline Thioredoxin Reductase $(\mathrm{TR}) \uparrow$ & 100 & 120 & ELISA & Yes & 108 \\
\hline
\end{tabular}

Note: $\downarrow / \uparrow$ Expression levels in PCa group compared to that of BPH group.

Abbreviations: ELISA, enzyme-linked immunosorbent assay, WB, Western blot, ELLA, enzyme-linked lectin assay, MS, mass spectrometry, FC, flow cytometry, IHC, immunohistochemistry, NMR, nuclear magnetic resonance, LC-MS, liquid chromatography-mass spectrometry, iTRAQ, Isobaric tag for relative and absolute quantitation. 
Table 3 Urine-Based Biomarkers Derived from Genomic Techniques

\begin{tabular}{|c|c|c|c|c|c|}
\hline Biomarkers & $\begin{array}{l}\text { BPH } \\
\text { (n) }\end{array}$ & $\begin{array}{l}\text { PCa } \\
\text { (n) }\end{array}$ & Method & $\begin{array}{l}\text { All } \\
\text { Significant } \\
(p<0.05)\end{array}$ & Reference \\
\hline 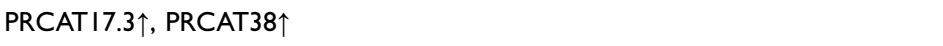 & 19 & 19 & qPCR & Yes & 109 \\
\hline 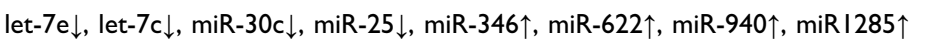 & 17 & 25 & qPCR & Yes & 110 \\
\hline PCA3个 & 26 & 22 & $\mathrm{qPCR}$ & Yes & 111 \\
\hline RASSFI $\uparrow$, GSTPI $\uparrow, R A R B \uparrow$ & 32 & 253 & $\mathrm{qPCR}$ & Yes & 112 \\
\hline miR-222-3p $\downarrow$, miR-24-3p $\downarrow$, miR-30c-5p $\uparrow$ & 29 & 215 & RT-PCR & Yes & 113 \\
\hline miR-222-3p $\downarrow$, miR-24-3p $\downarrow$, miR-30c-5p $\uparrow$ & 289 & 758 & RT-PCR & Yes & 114 \\
\hline 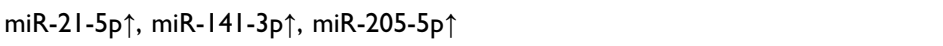 & 22 & 23 & $\mathrm{qPCR}$ & Yes & 115 \\
\hline 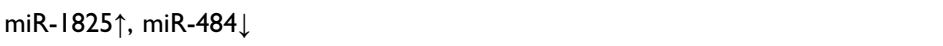 & 12 & 8 & WGS & Yes & 116 \\
\hline HISTIH4K & 29 & 57 & qPCR & No & 117 \\
\hline Prostate Cancer Antigen-3 (PCA3) $\uparrow$ & 40 & 24 & qPCR & Yes & 118 \\
\hline $\begin{array}{l}\text { UDP-N-Acetylglucosamine Pyrophosphorylase I (UAPI), PDZ and LIM Domain } 5 \\
\text { (PDLIM5), Inosine Monophosphate Dehydrogenase } 2 \text { (IMPDH2), Heat Shock } \\
\text { Protein Family D Member-I (HSPDI), Prostate Cancer Antigen-3 (PCA3), PSA, } \\
\text { Transmembrane Serine Protease } 2 \text { (TMPRSS2), ERG, Glyceraldehyde 3-Phosphate } \\
\text { Dehydrogenase (GAPDH), Beta-2-Microglobulin (B2M) }\end{array}$ & 60 & 61 & RT-PCR & Yes & 119 \\
\hline Prostate Cancer Antigen-3 (PCA3) $\uparrow, \mathrm{PSA} \uparrow$ & 26 & 70 & $\mathrm{qPCR}$ & Yes & 120 \\
\hline Whole-Genome Gene Expression & 24 & 25 & WGS & No & 121 \\
\hline Cadherin $3 \downarrow$ & 4 & 6 & WGS & Yes & 122 \\
\hline miR- $100 / 200 \mathrm{~b} \uparrow$ & 70 & 73 & qPCR & Yes & 123 \\
\hline $\operatorname{miR}-148 \mathrm{a} \uparrow, \mathrm{miR}-375 \uparrow$ & 23 & 215 & RT-PCR & Yes & 124 \\
\hline $\operatorname{miR}-21 \downarrow$ & 143 & 23 & qPCR & Yes & 125 \\
\hline 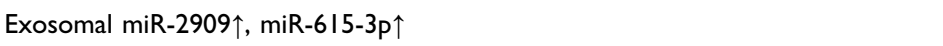 & 10 & 90 & $\mathrm{qPCR}$ & Yes & 126 \\
\hline SI00A $8 \downarrow$, SI00A9 $\downarrow$ & 363 & 283 & qPCR & Yes & 127 \\
\hline 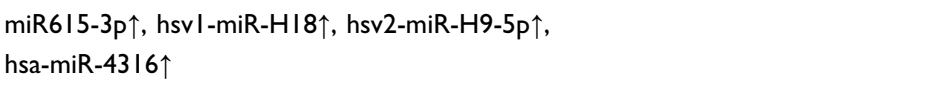 & 5 & 14 & $\begin{array}{l}\text { miRNA } \\
\text { Microarray }\end{array}$ & Yes & 128 \\
\hline PCA3/PSA ratio $\uparrow$ & 18 & 34 & RT-PCR & Yes & 129 \\
\hline
\end{tabular}

Note: $\downarrow / \uparrow$ Expression levels in PCa group compared to that of BPH group.

Abbreviations: Let-7, lethal-7 gene family, miRNA, microRNA, qPCR, quantitative polymerase chain reaction, RT-PCR, reverse transcription polymerase chain reaction, MS-PCR, methylation specific-polymerase chain reaction, WGS, whole genome sequencing, miRNA, microRNA.

must be practically and financially feasible, using approaches that return results in a timely manner.

The variety of biomarkers under investigation has inevitably led to the development of several commercially available prostate cancer tests that variously rely upon protein and gene expression measurements in biopsy, blood and urine samples. ${ }^{156}$ Each test utilizes a selection of different biomarkers, such as PSA-related measurements, expression of selected genes, the extent of gene methylation or detection of PCa-related gene fusions. Each test is positioned for clinical use in various cohorts of men at different stages of the disease management process, either before or after biopsy or following treatment. More tests are in development by 
Table 4 Urine-Based Biomarkers Derived from Proteomic Techniques

\begin{tabular}{|c|c|c|c|c|c|}
\hline Biomarkers & $\begin{array}{l}\text { BPH } \\
\text { (n) }\end{array}$ & $\begin{array}{l}\mathrm{PCa} \\
\text { (n) }\end{array}$ & Method & $\begin{array}{l}\text { All } \\
\text { Significant } \\
(p<0.05)\end{array}$ & Reference \\
\hline 48 Protein Groups & 9 & 9 & MS & Yes & 130 \\
\hline Glypican-I (GPC-I)^ & 37 & 41 & Immunoassay & Yes & 131 \\
\hline 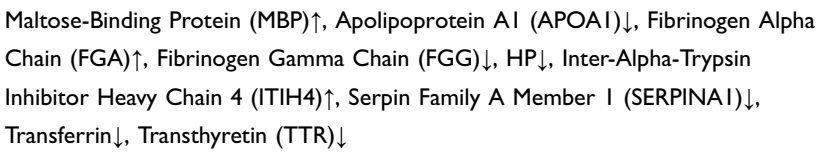 & 16 & 16 & 2-D DIGE, MS & Yes & 132 \\
\hline Matrix Metallopeptidase 9 (MMP-9) $\downarrow$ & 8 & 30 & Gelatin Zymography & Yes & 133 \\
\hline Fibronectin $\downarrow$, TP53INP2 $\downarrow$ & 12 & 8 & LC-MS/MS & Yes & 134 \\
\hline PSA Glycoforms & 61 & 38 & LC-MS/MS & Yes & 135 \\
\hline Saposin B $\downarrow$, Inter- $\alpha$-Trypsin Inhibitor Light Chain (ITIL) Fragments $\uparrow$ & 16 & 13 & $2 \mathrm{DE}, \mathrm{SS}$ & Yes & 136 \\
\hline Beta-2-Microglobulin (B2M) $\uparrow$, Pepsinogen $3 \uparrow$, and Mucin $3 \mathrm{~A} \uparrow$ & 83 & 90 & iTRAQ LC/LC/MS/MS & Yes & 137 \\
\hline PSA Glycoforms & 32 & 30 & LC-MS/MS & Yes & 138 \\
\hline $\begin{array}{l}\text { Furan } \uparrow \text {, 2-ethylhexanol } \downarrow \text {, 3,5-dimethylbenzaldehyde } \downarrow \text {, Santolin Triene } \downarrow \text {, } \\
\text { 2.6-dimethyl-7-octen-2-ol } \downarrow\end{array}$ & 21 & 29 & GC-MS & Yes & 139 \\
\hline 56 Intact N-glycopeptides & 6 & 6 & HILIC & Yes & 140 \\
\hline Fuc $\alpha$ |-6/3GlcNAc PSA Glycoforms $\uparrow$ & 15 & 16 & Immunoassay & Yes & 141 \\
\hline Survivin $\uparrow$ & 20 & 39 & ELISA, WB & Yes & 142 \\
\hline Osteopontin $\downarrow$, Prothrombin $\downarrow$ Peptides & 20 & 28 & LC-MS/MS & Yes & 143 \\
\hline Engrailed-2 (EN2) & 76 & 66 & ELISA & Yes & 144 \\
\hline Arginine $\uparrow$, Homoserine $\uparrow$, Proline $\uparrow$ & 50 & 50 & LC-MS/MS & Yes & 145 \\
\hline$\alpha-$ Methylacyl-CoA Racemase (AMACR), Hepsin & 76 & 66 & ELISA & No & 146 \\
\hline Spermine (Spm) $\uparrow$ & 88 & 66 & UPLC-MS/MS & Yes & 147 \\
\hline PSA Glycoforms & 93 & 74 & Capillary Electrophoresis & Yes & 148 \\
\hline Urinary Vesicle-Associated PSA Extraction Ratio $\uparrow$ & 122 & 85 & TEM & Yes & 149 \\
\hline Sarcosine/Creatinine Ratio $\uparrow$ & 208 & 209 & Sarcosine Oxidase Method & Yes & 150 \\
\hline PF $4 \mathrm{VI} \downarrow$, tPSA $\uparrow$, urinary CRISP $3 \uparrow$ & 48 & 86 & ITRAQ LC & Yes & 107 \\
\hline Ferritin-Creatinine Ratio $\uparrow$ & 3 & 3 & 2DE, MS, WB & Yes & 151 \\
\hline
\end{tabular}

Note: $\downarrow / \uparrow$ : Expression levels in PCa group compared to that of BPH group.

Abbreviations: MS, mass spectrometry, 2D DIGE, 2-dimensional fluorescence difference gel electrophoresis, LC-MS, liquid chromatography-mass spectrometry, ELISA, enzyme-linked immunosorbent assay, WB, Western blot, ELLA, enzyme-linked lectin assay, IHC, immunohistochemistry, iTRAQ, isobaric tag for relative and absolute quantitation, GC-MS, gas chromatography-mass spectrometry, HILIC, hydrophilic interaction liquid chromatography, UPLC-MS, ultra-performance liquid chromatographymass spectrometry, TEM, transmission electron microscopy.

various manufacturers and these will be on the market soon. However, there is no consensus about which of these tests is best and it is likely that multiple biomarkers must be considered by clinicians as part of the decision-making process for any given individual.

\section{Future Perspectives}

It is clear that there is a move towards the use of multiple biomarkers in a risk prediction model, which could include a combination of proteomic, genomic and clinical measurements. These multivariate models 
should result in a stronger degree of accuracy compared to that of a single marker for the prediction of $\mathrm{PCa}$. The standardized use of such risk stratification is recommended by the recent NICE guidelines. ${ }^{157}$ Several different models have been developed and are currently employed, each demonstrating their superiority to the use of tPSA measurement alone. The 4Kscore is a multivariate model that is used to identify the risk of aggressive PCa. Using a panel of 4 protein biomarkers, combined with clinical information, such as age and DRE results, the model achieved an AUC of 0.90 in one trial $(\mathrm{n}=1012) .{ }^{158}$ Similarly, the Stockholm-3 riskbased model $(\mathrm{S} 3 \mathrm{M})$ incorporates several plasma protein biomarkers (PSA, free PSA, intact PSA, hK2, MSMB, and $\mathrm{M} 1 \mathrm{C} 1)$ together with clinical data and individual genetic information to predict the likelihood of PCa. Using a validation cohort comprising of 47,688 men, the S3M was estimated to reduce the number of men biopsied by $53 \%$, as well as avoiding $76 \%$ of negative biopsies when compared to the standard tPSA method. ${ }^{159}$ Other risk stratification tools include the European Randomized Study of Screening for Prostate Cancer (ERSPC) risk calculator, which uses PSA, DRE, prostate volume and previous biopsy status to predict PCa risk. ${ }^{160}$ In North America, the Prostate Cancer Prevention Trial (PCPT) risk calculator uses PSA, DRE, family history, previous biopsy status, age, and race to do the same. ${ }^{161}$ Interestingly, one Irish study which directly compared these two models concluded the EPSRC was superior for the prediction of $\mathrm{PCa}$ in an Irish population $(n=2001)$ and advised combining it with the PHI model to improve accuracy. ${ }^{162}$ A study by Murphy et al in 2018 investigated a combination of data from DNA methylation, transcripts, protein and glycosylation biomarkers for use in a single PCa biomarker panel. Using modeling techniques on almost 200 variables the authors achieved an AUC of 0.91 when differentiating various stages of $\mathrm{PCa}$ severity from indolent to aggressive $(\mathrm{n}=158)$. This article is the first to incorporate data from five omic platforms retrieved from tissue and serum and clearly shows that the accuracy and predictive power of $\mathrm{PCa}$ models come from this multiplatform approach. ${ }^{163}$ Others have illustrated how technology can incorporate the measurements for ease of use. The Rotterdam Prostate Cancer Risk Calculator (RPCRC) utilizes a smartphone app with up to 11 clinical parameters used in combination to predict the risk of $\mathrm{PCa}$ and stratify those patients in need of a prostate biopsy. The algorithm has been used on around 6500 patients so far with AUC $0.72-0.81$ for the prediction of PCa. ${ }^{164}$ Although there is no consensus yet about which of these models is best, it is clear that many men presenting with symptoms of $\mathrm{PCa}$ could benefit from the use of these multivariate models to help determine their subsequent treatment.

Together, these studies demonstrate that the use of PSA screening can be improved to develop a more sophisticated method for the diagnosis and risk prediction of PCa. With such a vast majority of biomarkers being investigated, it is unfortunate that none have found clinical utility. The pathogenesis of $\mathrm{PCa}$ is complex and it is unlikely a single biomarker will arise as a replacement to PSA. Nonetheless, there is clear scope for further novel discoveries in this area, particularly in identifying new biomarkers that can be included in multivariate models that can aid PSA in identifying PCa patients earlier.

There is also an emerging role in the use of exosomes to aid in the diagnosis of PCa. Exosomes are small vesicular bodies released from a whole array of cells, they contain miRNA, mRNAs, and proteins that possess the potential to regulate signaling pathways in cells. ${ }^{165}$ Nilsson et al described in 2009 how they showed the presence of two known PCa biomarkers (PCA3 and TMPRAA2:ERG) within exosomes of urine patients, which in turn shows the potential for diagnosis and monitoring of patients in cancer care. ${ }^{166}$ One review states that exosomes have the potential of predicting the prognosis of castration-resistant $\mathrm{PCa}$, inducing $\mathrm{PCa}$ drug treatment sensitivity as well as being used as a marker for PCa drug response. In addition, exosomes can be used as a delivery vector to target malignant cells, as well as being utilized in tumor vaccination. ${ }^{165}$ However, with no gold standard in place for exosome isolation to validate these promising findings, it will not be possible until a uniformly defined method of isolation and characterization is in place. ${ }^{167}$ Nevertheless, with the accumulation of evidence, exosomes may prove to be a valuable source of biomarker material for informing $\mathrm{PCa}$ diagnosis, prognosis and therapy in the very near future.

\section{Summary}

Management of $\mathrm{PCa}$ in primary care presents several challenges for clinicians. Current diagnosis, based primarily on DRE and PSA measurements, can result in patients being sent for invasive biopsies that they do not require. A diagnostic test that can more accurately distinguish BPH 
from PCa would help alleviate clinical uncertainty in primary care, with tangible benefits for both the individual patients and the healthcare system. The use of multivariate biomarker measurement is required to differentiate $\mathrm{BPH}$ from $\mathrm{PCa}$. Combining selected biomarkers with clinical risk factors could be used to build a robust risk stratification model that could effectively triage patients within primary care; low and high risk. However, this clearly depends on the identification and validation of an optimal combination of biomarkers to use, so continued research is required to identify the combination of biomarkers with the sensitivity and specificity to direct clinical management.

\section{Disclosure}

Dr Mark W Ruddock is an employee of Randox Laboratories Ltd and holds no shares in the company. Mr Christopher McNally is a PhD student who Dr Ruddock jointly supervises with Dr Declan McKenna and Professor Tara Moore (both Ulster University). Dr Ruddock is the industrial supervisor at Randox Laboratories Ltd. Dr McKenna and Professor Moore are the academic supervisors at Ulster University. The $\mathrm{PhD}$ studentship is funded by the Randox-Ulster UniversityPhD Academy. The authors report no other conflicts of interest in this work.

\section{References}

1. Cooner WH, Mosley BR, Rutherford CL, et al. Prostate cancer detection in a clinical urological practice by ultrasonography, digital rectal examination and prostate specific antigen. $J$ Urol. 1990;143 (6):1146-1152. doi:10.1016/s0022-5347(17)40211-4

2. Malhotra A, Maughan D, Ansell J, et al. Choosing Wisely in the UK: the Academy of Medical Royal Colleges' initiative to reduce the harms of too much medicine. BMJ. 2015;350 (May):10-13. doi:10.1136/bmj.h2308

3. Chapple C. Medical treatment for benign prostatic hyperplasia. $B M J$. 1992;304(6836):1198-1199. doi:10.1136/bmj.304.6836.1198.

4. Skinder D, Zacharia I, Studin J, Covino J. Benign prostatic hyperplasia. J Am Acad Physician Assist. 2016;29(8):19-23. doi:10.1097/01.JAA.0000488689.58176.0a

5. McVary KT. BPH: epidemiology and comorbidities. Am J Manag Care. 2006;12(5 Suppl):S122-8.

6. Armenian HK, Lilienfeld AM, Diamond EL, Bross ID. Relation between benign prostatic hyperplasia and cancer of the prostate. A prospective and retrospective study. Lancet (London, England). 1974;2(7873):115-117. doi:10.1016/S0140-6736(74)91551-7

7. Chokkalingam AP, Nyrén O, Johansson J-E, et al. Prostate carcinoma risk subsequent to diagnosis of benign prostatic hyperplasia. Cancer. 2003;98 (8):1727-1734. doi:10.1002/cncr.11710

8. Schenk JM, Kristal AR, Arnold KB, et al. Association of symptomatic benign prostatic hyperplasia and prostate cancer: results from the prostate cancer prevention trial. Am J Epidemiol. 2011;173 (12):1419-1428. doi:10.1093/aje/kwq493

9. Dai X, Fang X, Ma Y, Xianyu J. Benign prostatic hyperplasia and the risk of prostate cancer and bladder cancer: a meta-analysis of observational studies. Medicine (Baltimore). 2016;95(18):e3493. doi:10.1097/ MD.0000000000003493
10. Hendriksen PJM, Dits NFJ, Kokame K, et al. Evolution of the androgen receptor pathway during progression of prostate cancer. Cancer Res. 2006;66(10):5012-5020. doi:10.1158/0008-5472. CAN-05-3082

11 Hammarsten J, Högstedt B. Clinical, haemodynamic, anthropometric, metabolic and insulin profile of men with high-stage and high-grade clinical prostate cancer. Blood Press. 2004;13 (1):47-55. doi:10.1080/08037050310025735.

12. Ørsted DD, Bojesen SE. The link between benign prostatic hyperplasia and prostate cancer. Nat Rev Urol. 2013;10(1):49-54. doi:10.1038/nrurol.2012.192

13. Alcaraz A, Hammerer P, Tubaro A, Schrö Der DFH, Castro R. Is there evidence of a relationship between benign prostatic hyperplasia and prostate cancer? Findings of a Literature Review. 2008. doi:10.1016/j.eururo.2008.11.011

14. Bostwick DG, Cooner WH, Denis L, Jones GW, Scardino PT, Murphy GP. The association of benign prostatic hyperplasia and cancer of the prostate. Cancer. 1992;70(S1):291-301. doi:10.1002/1097-0142(19920701)70:1+<291::AIDCNCR2820701317>3.0.CO;2-4

15. Gosselaar C, Kranse R, Roobol MJ, Roemeling S, Schröder FH. The interobserver variability of digital rectal examination in a large randomized trial for the screening of prostate cancer. Prostate. 2008;68(9):985-993. doi:10.1002/pros.20759

16. Alberts AR, Schoots IG, Roobol MJ. Prostate-specific antigen-based prostate cancer screening: past and future. Int J Urol. 2015;22(6):524-532. doi:10.1111/iju.12750

17. Duskova K, Vesely S. Prostate specific antigen. Current clinical application and future prospects. Biomed Pap Med Fac Univ Palacky Olomouc Czech Repub. 2015;159(1):18-26. doi: $10.5507 /$ bp.2014.046

18. Etzioni R, Gulati R, Cooperberg MR, Penson DM, Weiss NS, Thompson IM. Limitations of basing screening policies on screening trials: the US preventive services task force and prostate cancer screening. Med Care. 2013;51(4):295-300. doi:10.1097/MLR.0b013e31827da979

19. Draisma G, Boer R, Otto SJ, et al. Lead times and overdetection due to prostate-specific antigen screening: estimates from the European randomized study of screening for prostate cancer. $J$ Natl Cancer Inst. 2003;95(12):868-878. doi:10.1093/jnci/ 95.12.868

20. Catalona WJ, Hudson MA, Scardino PT, et al. Selection of optimal prostate specific antigen cutoffs for early detection of prostate cancer: receiver operating characteristic curves. J Urol. 1994;152 (6 Pt 1):2037-2042. doi:10.1016/S0022-5347(17)32300-5

21. De Angelis G, Rittenhouse HG, Mikolajczyk SD, Blair Shamel L, Semjonow A. Twenty years of PSA: from prostate antigen to tumor marker. Rev Urol. 2007;9(3):113-123.

22. Sokoll LJ, Sanda MG, Feng Z, et al. A prospective, multicenter, National Cancer Institute Early Detection Research Network study of [-2]proPSA: improving prostate cancer detection and correlating with cancer aggressiveness. Cancer Epidemiol Biomarkers Prev. 2010;19(5):1193-1200. doi:10.1158/1055-9965.EPI-10-0007

23. B V L, Griffin CR, Loeb S, et al. [-2]Proenzyme prostate specific antigen is more accurate than total and free prostate specific antigen in differentiating prostate cancer from benign disease in a prospective prostate cancer screening study. J Urol. 2010;183 (4):1355-1359. doi:10.1016/j.juro.2009.12.056

24. Morote J, Celma A, Planas J, et al. Eficacia del índice de salud prostática para identificar cánceres de próstata agresivos. Una validación institucional. Actas Urológicas Españolas. 2016;40 (6):378-385. doi:10.1016/j.acuro.2016.01.004

25. Nordström T, Akre O, Aly M, Grönberg H, Eklund M. Prostatespecific antigen (PSA) density in the diagnostic algorithm of prostate cancer. Prostate Cancer Prostatic Dis. 2018;21 (1):57-63. doi:10.1038/s41391-017-0024-7 
26. Verma A, St. Onge J, Dhillon K, Chorneyko A. PSA density improves prediction of prostate cancer. Can J Urol. 2014;21 (3):7312-7321. doi:10.1016/j.eururo.2014.08.027

27. Sebastianelli A, Morselli S, Vitelli FD, et al. The role of prostate-specific antigen density in men with low-risk prostate cancer suitable for active surveillance: results of a prospective observational study. Prostate Int. 2019;7(4):139-142. doi:10.1016/j.prnil.2019.02.001

28. Liu X, Tang J, Fei X, Li QY. Prostate-specific antigen (PSA) density and free to total PSA ratio in diagnosing prostate cancer with Prostate-specific antigen levels of $4.0 \mathrm{ng} / \mathrm{mL}$ or less. Iran J Public Health. 2015;44(11):1466-1472.

29. Vickers AJ, Till C, Tangen CM, Lilja H, Thompson IM. An empirical evaluation of guidelines on prostate-specific antigen velocity in prostate cancer detection. J Natl Cancer Inst. 2011;103(6):462-469. doi:10.1093/jnci/djr028

30. Hwang SI, Lee HJ. The future perspectives in transrectal prostate ultrasound guided biopsy. Prostate Int. 2014;2(4):153-160. doi:10.12954/PI.14062

31. NICE. Prostate Cancer: diagnosis and Treatment Contents.; 2014. Available from: https://www.nice.org.uk/guidance/cg175/ evidence/full-guideline-pdf-191710765. Accessed February 11, 2019.

32. Williamson DA, Barrett LK, Rogers BA, Freeman JT, Hadway P, Paterson DL. Infectious complications following transrectal ultrasound-guided prostate biopsy: new challenges in the era of multidrug-resistant Escherichia coli. Clin Infect Dis. 2013;57 (2):267-274. doi:10.1093/cid/cit193

33. Nazir B. Pain during transrectal ultrasound-guided prostate biopsy and the role of periprostatic nerve block: what radiologists should know. Korean $J$ Radiol Korean Soc Radiol. 2014; 15:543-553.

34. Nazir B. Pain during transrectal ultrasound-guided prostate biopsy and the role of periprostatic nerve block: what radiologists should know. Korean J Radiol. 2014;15(5):543-553. doi:10.3348/ kjr.2014.15.5.543

35. Otrock ZK, Oghlakian GO, Salamoun MM, Haddad M, Bizri ARN. Incidence of urinary tract infection following transrectal ultrasound guided prostate biopsy at a tertiary-care medical center in Lebanon. Infect Control Hosp Epidemiol. 2004;25 (10):873-877. doi:10.1086/502312

36. Nam RK, Saskin R, Lee Y, et al. Increasing hospital admission rates for urological complications after transrectal ultrasound guided prostate biopsy. J Urol. 2010;183(3):963-969. doi:10.1016/j.juro.2009.11.043

37. Nowak J, Malzahn U, Baur ADJ, et al. The value of ADC, T2 signal intensity, and a combination of both parameters to assess Gleason score and primary Gleason grades in patients with known prostate cancer. Acta Radiol. 2016;57(1):107-114. doi:10.1177/ 0284185114561915

38. Marcus DM, Rossi PJ, Nour SG, Jani AB. The impact of multiparametric pelvic magnetic resonance imaging on risk stratification in patients with localized prostate cancer. Urology. 2014;84 (1):132-137. doi:10.1016/j.urology.2014.03.012

39. Jie C, Rongbo L, Ping T. The value of diffusion-weighted imaging in the detection of prostate cancer: a meta-analysis. Eur Radiol. 2014;24(8):1929-1941. doi:10.1007/s00330-0143201-2

40. Lawrence EM, Gallagher FA, Barrett T, et al. Preoperative 3-T diffusion-weighted MRI for the qualitative and quantitative assessment of extracapsular extension in patients with intermediate- or high-risk prostate cancer. Am J Roentgenol. 2014;202(3):W280-W286. doi:10.2214/AJR.13.11754

41. Ghai S, Haider MA. Multiparametric-MRI in diagnosis of prostate cancer. Indian J Urol. 2015;31(3):194-201. doi:10.4103/09701591.159606
42. Baco E, Ukimura O, Rud E, et al. Magnetic resonance imaging-transectal ultrasound image-fusion biopsies accurately characterize the index tumor: correlation with step-sectioned radical prostatectomy specimens in 135 patients. Eur Urol. 2015;67 (4):787-794. doi:10.1016/j.eururo.2014.08.077

43. Le JD, Tan N, Shkolyar E, et al. Multifocality and prostate cancer detection by multiparametric magnetic resonance imaging: correlation with whole-mount histopathology. Eur Urol. 2015;67 (3):569-576. doi:10.1016/j.eururo.2014.08.079

44. Russo F, Regge D, Armando E, et al. Detection of prostate cancer index lesions with multiparametric magnetic resonance imaging (mp-MRI) using whole-mount histological sections as the reference standard. BJU Int. 2016;118(1):84-94. doi:10.1111/bju.13234

45. Strimbu K, Tavel JA. What are biomarkers? Curr Opin HIV AIDS. 2010;5(6):463. doi:10.1097/COH.0B013E32833ED177

46. Al-Kafaji G, Said H, Alam M, Al Naieb Z. Blood-based microRNAs as diagnostic biomarkers to discriminate localized prostate cancer from benign prostatic hyperplasia and allow cancer-risk stratification. Oncol Lett. 2018;16(1):1357-1365. doi:10.3892/ol.2018.8778

47. Al-Kafaji G, Al-Naieb ZT, Bakhiet M. Increased oncogenic microRNA-18a expression in the peripheral blood of patients with prostate cancer: a potential novel non-invasive biomarker. Oncol Lett. 2016;11(2):1201-1206. doi:10.3892/ ol.2015.4014

48. Cochetti G, Poli G, Guelfi G, Boni A, Egidi MG, Mearini E. Different levels of serum microRNAs in prostate cancer and benign prostatic hyperplasia: evaluation of potential diagnostic and prognostic role. Onco Targets Ther. 2016;9:7545-7553. doi:10.2147/OTT.S119027

49. Dumache R, Puiu M, Minciu R, et al. Retinoic acid receptor $\beta 2$ (RAR $\beta 2$ ): noninvasive biomarker for distinguishing malignant versus benign prostate lesions from bodily fluids. Chirurgia (Bucur). 107(6):780-784.

50. Feng J, Gang F, Li X, et al. Plasma cell-free DNA and its DNA integrity as biomarker to distinguish prostate cancer from benign prostatic hyperplasia in patients with increased serum prostate-specific antigen. Int Urol Nephrol. 2013;45(4):1023-1028. doi:10.1007/s11255-013-0491-2

51. Haldrup C, Kosaka N, Ochiya T, et al. Profiling of circulating microRNAs for prostate cancer biomarker discovery. Drug Deliv Transl Res. 2014;4(1):19-30. doi:10.1007/s13346-0130169-4

52. Kachakova D, Mitkova A, Popov E, et al. Combinations of serum prostate-specific antigen and plasma expression levels of let-7c, miR-30c, miR-141, and miR-375 as potential better diagnostic biomarkers for prostate cancer. DNA Cell Biol. 2015;34 (3):189-200. doi:10.1089/dna.2014.2663

53. Leidinger P, Hart M, Backes C, et al. Differential blood-based diagnosis between benign prostatic hyperplasia and prostate cancer: miRNA as source for biomarkers independent of PSA level, Gleason score, or TNM status. Tumour Biol. 2016;37 (8):10177-10185. doi:10.1007/s13277-016-4883-7

54. Mahn R, Heukamp LC, Rogenhofer S, von Ruecker A, Müller SC, Ellinger J. Circulating microRNAs (miRNA) in serum of patients with prostate cancer. Urology. 2011;77 (5):1265.e9-1265.e16. doi:10.1016/j.urology.2011.01.020

55. Minciu R, Dumache R, Gheorghe P, Daminescu L, Rogobete AF, Ionescu D. Molecular diagnostic of prostate cancer from body fluids using methylation-specific PCR (MS-PCR) method. Clin Lab. 2016;62(6):1183-1186.

56. Nikolić Z, Savić Pavićević D, Vučić N, et al. Assessment of association between genetic variants in microRNA genes hsa-miR-499, hsa-miR-196a2 and hsa-miR-27a and prostate cancer risk in Serbian population. Exp Mol Pathol. 2015;99 (1):145-150. doi:10.1016/j.yexmp.2015.06.009 
57. Reis IM, Ramachandran K, Speer C, Gordian E, Singal R. Serum GADD45a methylation is a useful biomarker to distinguish benign vs malignant prostate disease. Br J Cancer. 2015;113 (3):460-468. doi:10.1038/bjc. 2015.240

58. Sánchez BE, Aguayo A, Martínez B, et al. Using genetic and epigenetic markers to improve differential diagnosis of prostate cancer and benign prostatic hyperplasia by noninvasive methods in mexican patients. Clin Genitourin Cancer. 2018;16(4):e867e877. doi:10.1016/j.clgc.2018.02.004

59. Seyedolmohadessin SM, Akbari MT, Nourmohammadi Z, Basiri A, Pourmand G. Assessing the diagnostic value of plasma-free DNA in prostate cancer screening. Iran Biomed $J$. 2018;22(5):331-337. doi:10.29252/IBJ.22.5.331

60. Wach S, Al-Janabi O, Weigelt $\mathrm{K}$, et al. The combined serum levels of $m i R-375$ and urokinase plasminogen activator receptor are suggested as diagnostic and prognostic biomarkers in prostate cancer. Int $J$ Cancer. 2015;137(6):1406-1416. doi:10.1002/ ijc. 29505

61. Wang J, Ye H, Zhang D, et al. MicroRNA-410-5p as a potential serum biomarker for the diagnosis of prostate cancer. Cancer Cell Int. 2016;16(1):12. doi:10.1186/s12935-016-0285-6

62. Wang Y-H, Ji J, Wang B-C, et al. Tumor-derived exosomal long noncoding RNAs as promising diagnostic biomarkers for prostate cancer. Cell Physiol Biochem. 2018;46(2):532-545. doi:10.1159/ 000488620

63. Xue D, Zhou C-X, Shi Y-B, Lu H, He X-Z. MD-miniRNA could be a more accurate biomarker for prostate cancer screening compared with serum prostate-specific antigen level. Tumor Biol. 2015;36(5):3541-3547. doi:10.1007/s13277-014-2990-x

64. Zidan HE, Abdul-Maksoud RS, Elsayed WSH, Desoky EAM. Diagnostic and prognostic value of serum miR-15a and miR-161 expression among egyptian patients with prostate cancer. IUBMB Life. 2018;70(5):437-444. doi:10.1002/iub.1733

65. Adedapo K, Arinola O, Kareem O, Okolo C, Nwobi L, Shittu O. Diagnostic value of lipids, total antioxidants, and trace metals in benign prostate hyperplasia and prostate cancer. Niger J Clin Pract. 2012;15(3):293. doi:10.4103/1119-3077.100623

66. Amirrasouli H, Kazerouni F, Sanadizade M, et al. Accurate cut-off point for free to total prostate-specific antigen ratio used to improve differentiation of prostate cancer from benign prostate hyperplasia in Iranian population. Urol J. 2010;7(2):99-104.

67. Byrne JC, Downes MR, O'Donoghue N, et al. 2D-DIGE as a strategy to identify serum markers for the progression of prostate cancer. J Proteome Res. 2009;8(2):942-957. doi:10.1021/ pr800570s

68. Fan Y, Murphy TB, Byrne JC, Brennan L, Fitzpatrick JM, Watson RWG. Applying random forests to identify biomarker panels in serum 2D-DIGE data for the detection and staging of prostate cancer. J Proteome Res. 2011;10(3):1361-1373. doi: $10.1021 / \mathrm{pr} 1011069$

69. Cheng T, Li F, Wei R, et al. MMP26: A potential biomarker for prostate cancer. Curr Med Sci. 2017;37(6):891-894. doi:10.1007/ s11596-017-1823-8

70. Dai L, Li J, Ortega R, Qian W, Casiano CA, Zhang J-Y. Preferential autoimmune response in prostate cancer to cyclin B1 in a panel of tumor-associated antigens. J Immunol Res. 2014;2014:1-9. doi:10.1155/2014/827827

71. Ferrer-Batallé M, Llop E, Ramírez M, et al. Comparative study of blood-based biomarkers, $\alpha 2,3-$ Sialic Acid PSA and PHI, for highrisk prostate cancer detection. Int J Mol Sci. 2017;18(4):4. doi:10.3390/ijms 18040845

72. Fryczkowski M, Bułdak RJ, Hejmo T, Kukla M, ŻwirskaKorczala K. Circulating levels of omentin, Leptin, VEGF, and HGF and their clinical relevance with PSA marker in prostate cancer. Dis Markers. 2018;2018:1-9. doi:10.1155/2018/3852401
73. Fuchsova R, Topolcan O, Windrichova J, et al. PHI in the early detection of prostate cancer. Anticancer Res. 2015;35(9):4855-4857.

74. Fukushima K, Satoh T, Baba S, Yamashita K. 1,2-Fucosylated and - $\mathrm{N}$-acetylgalactosaminylated prostate-specific antigen as an efficient marker of prostatic cancer. Glycobiology. 2010;20 (4):452-460. doi:10.1093/glycob/cwp197

75. Grosman H, Fabre B, Lopez M, et al. Complex relationship between sex hormones, insulin resistance and leptin in men with and without prostatic disease. Aging Male. 2016;19(1):40-45. doi:10.3109/13685538.2015.1100600

76. Hagiwara K, Tobisawa Y, Kaya T, et al. Wisteria floribunda agglutinin and its reactive-glycan-carrying prostate-specific antigen as a novel diagnostic and prognostic marker of prostate cancer. Int J Mol Sci. 2017;18(2):261. doi:10.3390/ijms18020261

77. Jayapalan JJ, Ng KL, Razack AHA, Hashim OH. Identification of potential complementary serum biomarkers to differentiate prostate cancer from benign prostatic hyperplasia using gel- and lectin-based proteomics analyses. Electrophoresis. 2012;33 (12):1855-1862. doi:10.1002/elps.201100608

78. Jokerst JV, Chen Z, Xu L, et al. A magnetic bead-based sensor for the quantification of multiple prostate cancer biomarkers. PLoS One. 2015;10(9):e0139484. doi:10.1371/journal.pone.0139484

79. Kaynar M, Yildirim ME, Gul M, Kilic O, Ceylan K, Goktas S. Benign prostatic hyperplasia and prostate cancer differentiation via platelet to lymphocyte ratio. Cancer Biomarkers. 2015;15 (3):317-323. doi:10.3233/CBM-150458

80. Kucukdurmaz F, Efe E, Çelik A, Dagli H, Kılınc M, Resim S. Evaluation of serum prolidase activity and oxidative stress markers in men with BPH and prostate cancer. BMC Urol. 2017;17 (1):116. doi:10.1186/s12894-017-0303-6

81. Leidinger P, Keller A, Milchram L, et al. Combination of autoantibody signature with PSA level enables a highly accurate blood-based differentiation of prostate cancer patients from patients with benign prostatic Hyperplasia. Saleem M, ed. PLoS One. 2015;10(6):e0128235. doi:10.1371/journal.pone.0128235

82. Levin RA, Lund ME, Truong Q, et al. Development of a reliable assay to measure glypican-1 in plasma and serum reveals circulating glypican-1 as a novel prostate cancer biomarker. Oncotarget. 2018;9 (32):22359-22367. doi:10.18632/oncotarget.25009

83. El Melegy NT, Aboulella HA, Abul-Fadl AM, Mohamed NA. Potential biomarkers for differentiation of benign prostatic hyperplasia and prostate cancer. Br J Biomed Sci. 2010;67(3):109-112. doi:10.1080/09674845.2010.11730306

84. Menschikowski M, Hagelgans A, Fuessel S, et al. Serum amyloid A, phospholipase A2-IIA and C-reactive protein as inflammatory biomarkers for prostate diseases. Inflamm Res. 2013;62 (12):1063-1072. doi:10.1007/s00011-013-0665-5

85. Menschikowski M, Hagelgans A, Fuessel S, et al. Serum levels of secreted group IIA Phospholipase A2 in benign prostatic hyperplasia and prostate cancer: a biomarker for inflammation or neoplasia? Inflammation. 2012;35(3):1113-1118. doi:10.1007/ s10753-011-9418-1

86. Mhatre DR, Mahale SD, Khatkhatay MI, et al. Development of an ELISA for sPSP94 and utility of the sPSP94/SPSA ratio as a diagnostic indicator to differentiate between benign prostatic hyperplasia and prostate cancer. Clin Chim Acta. 2014;436:256-262. doi:10.1016/J.CCA.2014.06.006

87. Michalakis K, Ilias I, Triantafyllou A, et al. Detection of prostate cancer by sialic acid level in patients with non-diagnostic levels of prostate-specific antigen. Maturitas. 2012;73(4):325-330. doi:10.1016/j.maturitas.2012.07.016

88. Dwek MV, Jenks A, Leathem AJC. A sensitive assay to measure biomarker glycosylation demonstrates increased fucosylation of prostate specific antigen (PSA) in patients with prostate cancer compared with benign prostatic hyperplasia. Clin Chim Acta. 2010;411(23-24):1935-1939. doi:10.1016/J.CCA.2010.08.009 
89. Miyoshi Y, Uemura H, Suzuki K, et al. High serum dihydrotestosterone examined by ultrasensitive LC-MS/MS as a predictor of benign prostatic hyperplasia or Gleason score 6 cancer in men with prostate-specific antigen levels of 3-10 ng/mL. Andrology. 2017;5(2):262-267. doi:10.1111/andr.12294

90. O'Rourke DJ, DiJohnson DA, Caiazzo RJ, et al. Autoantibody signatures as biomarkers to distinguish prostate cancer from benign prostatic hyperplasia in patients with increased serum prostate specific antigen. Clin Chim Acta. 2012;413(5-6):561-567. doi:10.1016/J.CCA.2011.11.027

91. Orywal K, Jelski W, Werel T, Szmitkowski M. The diagnostic significance of serum alcohol dehydrogenase isoenzymes and aldehyde dehydrogenase activity in prostate cancer patients. Anticancer Res. 2017;37(9):4961-4965. doi:10.21873/anticanres.11906

92. Pace G, Massimo Di C, De Amicis D, Vicentini C, Ciancarelli MGT. Inflammation and endothelial activation in benign prostatic hyperplasia and prostate cancer. Int Braz J Urol. 2011;37(5):617-622. doi:10.1590/S167755382011000500008

93. Peehl DM, Chen Z, Nolley R. Serum Mac-2BP does not distinguish men with high grade, large volume prostate cancer from men with benign prostatic hyperplasia. Prostate. 2011;71 (1):26-31. doi:10.1002/pros.21218

94. Rainato G, Fabricio ASC, Zancan M, et al. Evaluating serum insulin-like growth factor 1 and insulin-like growth factor binding protein 3 as markers in prostate cancer diagnosis. Int $J$ Biol Markers. 2016;31(3):317-323. doi:10.5301/jbm.5000200

95. Ravipaty $\mathrm{S}, \mathrm{Wu} \mathrm{W}, \mathrm{Dalvi} \mathrm{A}$, et al. Clinical validation of a serum protein panel (FLNA, FLNB and KRT19) for diagnosis of prostate cancer. J Mol Biomark Diagn. 2017;8(02):2. doi:10.4172/ 2155-9929.1000323

96. Rehman I, Evans CA, Glen A, et al. iTRAQ identification of candidate serum biomarkers associated with metastatic progression of human prostate cancer. PLoS One. 2012;7(2):e30885. doi:10.1371/journal.pone.0030885

97. Sarrats A, Comet J, TabarÃ@s G, et al. Differential percentage of serum prostate-specific antigen subforms suggests a new way to improve prostate cancer diagnosis. Prostate. 2010;70(1):1-9. doi:10.1002/pros.21031

98. Sarwar S, Adil MAM, Nyamath P, Ishaq M. Biomarkers of prostatic cancer: an attempt to categorize patients into prostatic carcinoma, benign prostatic hyperplasia, or prostatitis based on serum prostate specific antigen, prostatic acid phosphatase, calcium, and phosphorus. Prostate Cancer. 2017;2017:5687212. doi: $10.1155 / 2017 / 5687212$

99. Sharif MR, Nikoueinejad H, Shaabani A, Mahmoudi H, Akbari H, Einollahi B. Association of the serum vascular endothelial growth factor levels with benign prostate hyperplasia and prostate malignancies. Nephrourol Mon. 2014;6(3):e14778. doi:10.5812/numonthly. 14778

100. Fu S, Zhang X, Niu Y, Wang R-T. Prostate specific antigen, mean platelet volume, and platelet distribution width in combination to discriminate prostatecancer from benign prostate hyperplasia. Asian Pac J Cancer Prev. 2018;19(3):699-702. doi:10.22034/ APJCP.2018.19.3.699

101. Silva PMS, Lima ALR, Silva BVM, Coelho LCBB, Dutra RF, Correia MTS. Cratylia mollis lectin nanoelectrode for differential diagnostic of prostate cancer and benign prostatic hyperplasia based on label-free detection. Biosens Bioelectron. 2016;85:171-177. doi:10.1016/j.bios.2016.05.004

102. Uyeturk U, Sarıcı H, Kin Tekce B, et al. Serum omentin level in patients with prostate cancer. Med Oncol. 2014;31(4):923. doi:10.1007/s12032-014-0923-6

103. Wang L, Ma L, Wang X, Li B, Guo S, Qiao Q. Association of serum EPCA-2 level with prostate cancer in Chinese Han population. Int J Clin Exp Pathol. 2015;8(8):9397-9403.
104. Wang X, An P, Zeng J, et al. Serum ferritin in combination with prostate-specific antigen improves predictive accuracy for prostate cancer. Oncotarget. 2017;8(11):17862-17872. doi:10.18632/ oncotarget. 14977

105. Worst TS, von Hardenberg J, Gross JC, et al. Databaseaugmented mass spectrometry analysis of exosomes identifies Claudin 3 as a putative prostate cancer biomarker. $\mathrm{Mol}$ Cell Proteomics. 2017;16(6):998-1008. doi:10.1074/mcp. M117.068577

106. $\mathrm{Xu} \mathrm{L}, \mathrm{Hu} \mathrm{X}, \mathrm{Zhu} \mathrm{Y}$, et al. Additional value of the ratio of serum total testosterone to total prostate-specific antigen in the diagnosis of prostate cancer in a Chinese population. Andrologia. 2018;50 (2):e12872. doi:10.1111/and.12872

107. Zhang M, Chen L, Yuan Z, et al. Combined serum and EPS-urine proteomic analysis using iTRAQ technology for discovery of potential prostate cancer biomarkers. Discov Med. 2016;22 (122):281-295.

108. Zhang W, Zheng X, Wang X. Oxidative stress measured by thioredoxin reductase level as potential biomarker for prostate cancer. Am J Cancer Res. 2015;5(9):2788-2798.

109. Bayat H, Narouie B, Ziaee S-AM, Mowla SJ. Two long non-coding RNAs, Prcat17.3 and Prcat38, could efficiently discriminate benign prostate hyperplasia from prostate cancer. Prostate. 2018;78(11):812-818. doi:10.1002/pros.23538

110. Chen Z-H, Zhang G-L, Li H-R, et al. A panel of five circulating microRNAs as potential biomarkers for prostate cancer. Prostate. 2012;72(13):1443-1452. doi:10.1002/pros.22495

111. Fonseca Coelho F, Loli Guimarães F, Ribeiro Cabral WL, et al. Expression of PCA3 and PSA genes as a biomarker for differential diagnosis of nodular hyperplasia and prostate cancer. Genet $\mathrm{Mol}$ Res. 2015;14(4):13519-13531. doi:10.4238/2015.October.28.13

112. Daniunaite K, Jarmalaite S, Kalinauskaite N, et al. Prognostic value of RASSF1 promoter methylation in prostate cancer. J Urol. 2014;192(6):1849-1855. doi:10.1016/j.juro.2014.06.075

113. Fredsøe J, Rasmussen AKI, Thomsen AR, et al. Diagnostic and prognostic microRNA biomarkers for prostate cancer in cell-free urine. Eur Urol Focus. 2018;4(6):825-833. doi:10.1016/j. euf.2017.02.018

114. Fredsøe J, Rasmussen AKI, Laursen EB, et al. Independent validation of a diagnostic noninvasive 3-MicroRNA Ratio Model $(u \mathrm{CaP})$ for prostate cancer in cell-free urine. Clin Chem. 2019;65(4):540-548. doi:10.1373/clinchem.2018.296681

115. Ghorbanmehr N, Gharbi S, Korsching E, Tavallaei M, Einollahi B, Mowla SJ. miR-21-5p, miR-141-3p, and miR-205$5 \mathrm{p}$ levels in urine-promising biomarkers for the identification of prostate and bladder cancer. Prostate. 2019;79(1):88-95. doi:10.1002/pros. 23714

116. Haj-Ahmad TA, Abdalla MA, Haj-Ahmad Y. Potential urinary miRNA biomarker candidates for the accurate detection of prostate cancer among benign prostatic hyperplasia patients. J Cancer. 2014;5(3):182-191. doi:10.7150/jca.6799

117. Kachakova D, Mitkova A, Popov E, et al. Evaluation of the clinical value of the newly identified urine biomarker HIST1H4K for diagnosis and prognosis of prostate cancer in Bulgarian patients. J BUON. 18;3:660-668.

118. Li M, Zhou D, Zhang W, Gao S, Zhou X. Urine PCA3 mRNA level in diagnostic of prostate cancer. J Cancer Res Ther. 2018;14 (4):864. doi:10.4103/jcrt.JCRT_734_17

119. Ma W, Diep K, Fritsche HA, Shore N, Albitar M. Diagnostic and prognostic scoring system for prostate cancer using urine and plasma biomarkers. Genet Test Mol Biomarkers. 2014;18 (3):156-163. doi:10.1089/gtmb.2013.0424

120. Mearini E, Antognelli C, Del Buono C, et al. The combination of urine DD3 ${ }^{\text {PCA3 }}$ mRNA and PSA mRNA as molecular markers of prostate cancer. Biomarkers. 2009;14(4):235-243. doi:10.1080/ 13547500902807306 
121. Özdemir TR, Şimşir A, Onay H, Cüreklibatır İ, Özkınay F, Akın H. Whole-genome gene expression analysis in urine samples of patients with prostate cancer and benign prostate hyperplasia. Urol Oncol Semin Orig Investig. 2017;35(10):607. e15-607.e24. doi:10.1016/j.urolonc.2017.05.020

122. Royo F, Zuñiga-Garcia P, Torrano V, et al. Transcriptomic profiling of urine extracellular vesicles reveals alterations of $\mathrm{CDH} 3$ in prostate cancer. Oncotarget. 2016;7(6):6835-6846. doi:10.18632/ oncotarget.6899

123. SALIDO-GUADARRAMA AI, MORALES-MONTOR JG, RANGEL-ESCAREÑO C, et al. Urinary microRNA-based signature improves accuracy of detection of clinically relevant prostate cancer within the prostate-specific antigen grey zone. Mol Med Rep. 2016;13(6):4549-4560. doi:10.3892/mmr.2016.5095

124. Stuopelytė K, Daniūnaite K, Jankevičius F, Jarmalaitė S. Detection of miRNAs in urine of prostate cancer patients. Medicina (B Aires). 2016;52(2):116-124. doi:10.1016/J.MEDICI.2016.02.007

125. Stuopelyte K, Daniunaite K, Bakavicius A, Lazutka JR, Jankevicius F, Jarmalaite $\mathrm{S}$. The utility of urine-circulating miRNAs for detection of prostate cancer. Br J Cancer. 2016;115 (6):707-715. doi:10.1038/bjc.2016.233

126. Wani S, Kaul D, Mavuduru RS, Kakkar N, Bhatia A. Urinaryexosomal miR-2909: a novel pathognomonic trait of prostate cancer severity. J Biotechnol. 2017;259:135-139. doi:10.1016/J. JBIOTEC.2017.07.029

127. Yun SJ, Yan C, Jeong P, et al. Comparison of mRNA, protein, and urinary nucleic acid levels of S100A8 and S100A9 between Prostate Cancer and BPH. Ann Surg Oncol. 2015;22 (7):2439-2445. doi:10.1245/s10434-014-4194-4

128. Yun SJ, Jeong P, Kang HW, et al. Urinary MicroRNAs of prostate cancer: virus-Encoded hsv1-miRH18 and hsv2-miR-H9-5p could be valuable diagnostic markers. Int Neurourol J. 2015;19 (2):74-84. doi:10.5213/inj.2015.19.2.74

129. Zheng K, Dou Y, He L, et al. Improved sensitivity and specificity for prostate cancer diagnosis based on the urine PCA3/PSA ratio acquired by sequence-specific RNA capture. Oncol Rep. 2015;34 (5):2439-2444. doi:10.3892/or.2015.4266

130. Adeola HA, Soares NC, Paccez JD, Kaestner L, Blackburn JM, Zerbini LF. Discovery of novel candidate urinary protein biomarkers for prostate cancer in a multiethnic cohort of South African patients via label-free mass spectrometry. PROTEOMICS - Clin Appl. 2015;9(5-6):597-609. doi:10.1002/prca.201400197

131. Campbell DH, Lund ME, Nocon AL, et al. Detection of glypican-1 (GPC-1) expression in urine cell sediments in prostate cancer. Pizzo S V, ed. PLoS One. 2018;13(4):e0196017. doi:10.1371/journal.pone.0196017

132. Davalieva K, Kiprijanovska S, Komina S, Petrusevska G, Zografska NC, Polenakovic M. Proteomics analysis of urine reveals acute phase response proteins as candidate diagnostic biomarkers for prostate cancer. Proteome Sci. 2015;13(1):2. doi:10.1186/s12953-014-0059-9

133. Macchia A, Mariano A, Terracciano D, et al. Matrix metalloproteinase- 2 and -9 in the urine of prostate cancer patients. Oncol Rep. 2010;24(1):3-8. doi:10.3892/or_00000821

134. Haj-Ahmad TA, Abdalla MA, Haj-Ahmad Y. Potential urinary protein biomarker candidates for the accurate detection of prostate cancer among benign prostatic hyperplasia patients. $J$ Cancer. 2014;5(2):103-114. doi:10.7150/jca.6890

135. Hsiao C-J, Tzai T-S, Chen C-H, Yang W-H, Chen C-H. Analysis of urinary prostate-specific antigen glycoforms in samples of prostate cancer and benign prostate hyperplasia. Dis Markers. 2016;2016:8915809. doi:10.1155/2016/8915809
136. Jayapalan JJ, Ng KL, Shuib AS, Razack AHA, Hashim OH. Urine of patients with early prostate cancer contains lower levels of light chain fragments of inter-alpha-trypsin inhibitor and sapo$\sin \mathrm{B}$ but increased expression of an inter-alpha-trypsin inhibitor heavy chain 4 fragment. Electrophoresis. 2013;34 (11):1663-1669. doi:10.1002/elps.201200583

137. Jedinak A, Curatolo A, Zurakowski D, et al. Novel non-invasive biomarkers that distinguish between benign prostate hyperplasia and prostate cancer. BMC Cancer. 2015;15(1):259. doi:10.1186/ s12885-015-1284-z

138. Jia G, Dong Z, Sun C, et al. Alterations in expressed prostate secretion-urine PSA N-glycosylation discriminate prostate cancer from benign prostate hyperplasia. Oncotarget. 2017;8 (44):76987-76999. doi:10.18632/oncotarget.20299

139. Jiménez-Pacheco A, Salinero-Bachiller M, Iribar MC, LópezLuque A, Miján-Ortiz JL, Peinado JM. Furan and p-xylene as candidate biomarkers for prostate cancer. Urol Oncol Semin Orig Investig. 2018;36(5):243.e21-243.e27. doi:10.1016/j.urolonc. 2017.12.026

140. Kawahara R, Ortega F, Rosa-Fernandes L, et al. Distinct urinary glycoprotein signatures in prostate cancer patients. Oncotarget. 2018;9(69):33077-33097. doi:10.18632/oncotarget.26005

141. Kekki H, Peltola M, van Vliet S, Bangma C, van Kooyk Y, Pettersson K. Improved cancer specificity in PSA assay using Aleuria aurantia lectin coated Eu-nanoparticles for detection. Clin Biochem. 2017;50(1-2):54-61. doi:10.1016/J. CLINBIOCHEM.2016.06.015

142. Khan S, Jutzy JMS, Valenzuela MMA, et al. Plasma-derived exosomal survivin, a plausible biomarker for early detection of prostate cancer. Li J, ed. PLoS One. 2012;7(10):e46737. doi:10.1371/journal.pone.0046737

143. Li C, Zang T, Wrobel K, Huang -JT-J, Nabi G. Quantitative urinary proteomics using stable isotope labelling by peptide dimethylation in patients with prostate cancer. Anal Bioanal Chem. 2015;407(12):3393-3404. doi:10.1007/s00216-015-8569-6

144. Marszałł MP, Sroka W, Adamowski M, et al. Engrailed-2 protein as a potential urinary prostate cancer biomarker. Eur J Cancer Prev. 2015;24(1):51-56. doi:10.1097/CEJ.0000000000000046

145. Sroka WD, Boughton BA, Reddy P, et al. Determination of amino acids in urine of patients with prostate cancer and benign prostate growth. Eur J Cancer Prev. 2017;26(2):131-134. doi:10.1097/ CEJ.0000000000000248

146. Sroka WD, Adamowski M, Słupski P, et al. Alpha-methylacylCoA racemase and hepsin as urinary prostate cancer markers. Int J Biol Markers. 2015;30(4):401-406. doi:10.5301/ jbm.5000146

147. Tsoi T-H, Chan C-F, Chan W-L, et al. Urinary polyamines: a pilot study on their roles as prostate cancer detection biomarkers. Amendola R, ed. PLoS One. 2016;11(9):e0162217. doi:10.1371/ journal.pone. 0162217

148. Vermassen T, Van Praet C, Lumen N, et al. Urinary prostate protein glycosylation profiling as a diagnostic biomarker for prostate cancer. Prostate. 2015;75(3):314-322. doi:10.1002/ pros. 22918

149. Vermassen T, D'Herde K, Jacobus D, et al. Release of urinary extracellular vesicles in prostate cancer is associated with altered urinary N-glycosylation profile. J Clin Pathol. 2017;70 (10):838-846. doi:10.1136/jclinpath-2016-204312

150. Wang M, Zou L, Liang J, et al. The urinary sarcosine/creatinine ratio is a potential diagnostic and prognostic marker in prostate cancer. Med Sci Monit. 2018;24:3034-3041. doi:10.12659/ MSM.909949 
151. Zhao H, Zhao X, Lei T, Zhang M. Screening, identification of prostate cancer urinary biomarkers and verification of important spots. Invest New Drugs. 2019;37(5):935-947. doi:10.1007/ s10637-018-0709-3

152. Hashim $\mathrm{OH}$, Jayapalan JJ, Lee CS. Lectins: an effective tool for screening of potential cancer biomarkers. PeerJ. 2017;2017:9. doi:10.7717/peerj.3784

153. Narain NR, Diers AR, Lee A, et al. Identification of Filamin-A and -B as potential biomarkers for prostate cancer. Futur Sci OA. 2017;3(1):FSO161. doi:10.4155/fsoa-2016-0065

154. Di Donato M, Cernera G, Auricchio F, Migliaccio A, Castoria G. Cross-talk between androgen receptor and nerve growth factor receptor in prostate cancer cells: implications for a new therapeutic approach. Cell Death Discov. 2018;4(1):1-2. doi:10.1038/ s41420-017-0024-3

155. Wu D, Ni J, Beretov $\mathrm{J}$, et al. Urinary biomarkers in prostate cancer detection and monitoring progression. Crit Rev Oncol Hematol. 2017;118:15-26. doi:10.1016/j.critrevonc.2017.08.002

156. Alford AV, Brito JM, Yadav KK, Yadav SS, Tewari AK, Renzulli J. The use of biomarkers in prostate cancer screening and treatment. Rev Urol. 2017;19(4):221-234. doi:10.3909/riu0772

157. Overview | Prostate cancer: diagnosis and management Guidance | NICE. Available from: https://www.nice.org.uk/gui dance/ng131. 2019. Accessed December 12, 2019.

158. Punnen S, Pavan N, Parekh DJ. Finding the wolf in sheep's clothing: the $4 \mathrm{Kscore}$ is a novel blood test that can accurately identify the risk of aggressive prostate cancer. Rev Urol. 2015;17(1):3-13.

159. Eklund M, Nordström T, Aly M, et al. The Stockholm-3 (STHLM3) model can improve prostate cancer diagnostics in men aged 50-69 yr compared with current prostate cancer testing. Eur Urol Focus. 2018;4(5):707-710. doi:10.1016/j.euf.2016.10.009
160. Schröder FH, Hugosson J, Roobol MJ, et al. Screening and prostate-cancer mortality in a randomized european study. $N$ Engl J Med. 2009;360(13):1320-1328. doi:10.1056/NEJMoa0810084

161. Thompson IM, Ankerst DP, Chi C, et al. Assessing prostate cancer risk: results from the prostate cancer prevention trial. J Natl Cancer Inst. 2006;98(8):529-534. doi:10.1093/jnci/djj131

162. Foley RW, Maweni RM, Gorman L, et al. European Randomised Study of Screening for Prostate Cancer (ERSPC) risk calculators significantly outperform the Prostate Cancer Prevention Trial (PCPT) 2.0 in the prediction of prostate cancer: a multi-institutional study. BJU Int. 2016;118(5):706-713. doi:10.1111/bju.13437

163. Murphy K, Murphy BT, Boyce S, et al. Integrating biomarkers across omic platforms: an approach to improve stratification of patients with indolent and aggressive prostate cancer. Mol Oncol. 2018;12(9):1513-1525. doi:10.1002/1878-0261.12348

164. Pereira-Azevedo N, Osório L, Fraga A, Roobol MJ. Rotterdam prostate cancer risk calculator: development and usability testing of the mobile phone app. JMIR Cancer. 2017;3(1):e1. doi:10.2196/cancer.6750

165. Pan J, Ding M, Xu K, Yang C, Mao LJ. Exosomes in diagnosis and therapy of prostate cancer. Oncotarget. 2017;8 (57):97693-97700. doi:10.18632/oncotarget.18532

166. Nilsson J, Skog J, Nordstrand A, et al. Prostate cancer-derived urine exosomes: a novel approach to biomarkers for prostate cancer. $\mathrm{Br}$ J Cancer. 2009;100(10):1603-1607. doi:10.1038/sj.bjc.6605058

167. Ludwig N, Whiteside TL, Reichert TE. Challenges in exosome isolation and analysis in health and disease. Int J Mol Sci. 2019;20(19):19. doi:10.3390/ijms20194684

\section{Publish your work in this journal}

Cancer Management and Research is an international, peer-reviewed open access journal focusing on cancer research and the optimal use of preventative and integrated treatment interventions to achieve improved outcomes, enhanced survival and quality of life for the cancer patient.
The manuscript management system is completely online and includes a very quick and fair peer-review system, which is all easy to use. Visit http://www.dovepress.com/testimonials.php to read real quotes from published authors. 